\section{CONVERSION OF MAGNETIC FIELD ENERGY INTO KINETIC ENERGY IN THE SOLAR WIND}

\author{
Y. C. Whang
}

\begin{abstract}
The outflow of the solar magnetic field energy (the radial component of the Poynting vector) per steradian is inversely proportional to the solar wind velocity. It is a decreasing function of the heliocentric distance. When the magnetic field effect is included in the one-fluid model of the solar wind, the transformation of magnetic field energy into kinetic energy during the expansion process increases the solar wind velocity at $1 \mathrm{AU}$ by 17 percent. The predicted solar wind conditions at $1 \mathrm{AU}$ are $u=302 \mathrm{~km} / \mathrm{sec}$, $n=8$ protons $/ \mathrm{cm}^{3}, T=1.5 \times 10^{5}{ }^{\circ} \mathrm{K}, q=1.4 \times 10^{-2} \mathrm{erg} / \mathrm{cm}^{2} / \mathrm{sec}, B=7.3 \gamma \phi=129.5^{\circ}$, and $\beta=1.58$. They agree very well with the observed quiet solar wind.
\end{abstract}

\section{INTRODUCTION}

Since the original application of hydrodynamic equations to the solar wind by Parker [1958], a number of theoretical models have been developed to study the solar wind, notably the one-fluid models studied by Noble and Scarf [1963] and by Whang and Chang [1965], and the two-fluid model by Sturrock and Hartle [1966] and by Hartle and Sturrock [1968]. The one-fluid model assumed that all types of particles (electrons, proton, helium, etc.) have the same temperature, while the two-fluid model took into consideration the different temperatures for protons and electrons. The inviscid model of Whang and Chang predicted a solar wind velocity of $260 \mathrm{~km} / \mathrm{sec}$ at $1 \mathrm{AU}$. The solution of Hartle and Sturrock predicted a velocity of $250 \mathrm{~km} / \mathrm{sec}$ at $1 \mathrm{AU}$. These velocites are about 20 percent below the observed quiet solar wind condition [Hundhausen, 1970].

The interplanetary magnetic field has a strong coupling with the dynamics of the solar wind. The magnetic field organizes the collisionless plasma into a continuum in the expansion process of the solar wind. It is well known that when the solar wind flows across a shock wave, the

The author is with the Department of Aerospace and Atmospheric Sciences, The Catholic University of America, Washington, D. C. magnetic field energy flux and the convective thermal energy increase during the compression process at the expense of the kinetic energy. In a reverse process, during the magnetohydrodynamic expansion of the solar wind, the magnetic field energy as well as the thermal energy is continuously converted into kinetic energy. Therefore, we propose that in a magnetohydrodynamic model of the solar wind, which includes an additional energy source, the predicted solar wind velocity can be increased closer to the observed quiet condition.

Various mechanisms for the additional energy source needed to increase the solar wind velocity have been proposed by many authors. Barnes [1968, 1969] proposed that a significant amount of energy is carried by the hydromagnetic waves. The dissipation of hydromagnetic waves adds its energy into the solar wind flow. Jokipii and Davis [1969] proposed that the observed long-lived velocity streams are the source of long-wavelength turbulence, which in turn serves as an efficient and variable heat source for the solar plasma. In this paper, we will show that the magnetic field energy flow per steradian is a decreasing function of the heliocentric distance $r$. The total decrease of field energy flow from $r=2 \mathrm{r}_{\odot}$ to $r=1 \mathrm{AU}$ is about 25 percent of the convective kinetic energy at $1 \mathrm{AU}$. Therefore, during the expansion process of the solar wind, the conversion of 
field energy into kinetic energy increases the solar wind velocity by 17 percent.

The solution of a steady-state magnetohydrodynamic one-fluid model of the solar wind is carried out in this paper. The model assumes that the solar wind velocity is in radial directions away from the center of the sun, and its temperature is isotropic. The solar wind conditions near the equatorial plane predicted by the present solution clearly demonstrate the process of energy conversion in the solar wind on a quantitative basis.

\section{DECREASE OF THE MAGNETIC FIELD ENERGY FLOW}

Spiral Pattern of the Magnetic Field

The steady-state solar magnetic field is governed by Maxwell's equations

$$
\begin{array}{r}
\nabla \cdot \mathbf{B}=0 \\
\nabla \times(\mathbf{u} \times \mathbf{B})=0
\end{array}
$$

Spherical coordinates $(r, \psi, \omega)$ are used in this paper; $\psi$ denotes the colatitude measured from the north pole of the sun. If $\mathbf{B}=\mathrm{e}_{r} B_{r}(r, \psi)+\mathrm{e}_{\omega} B \omega(r, \psi)$, then we can write equations (1) and (2) as

$$
\begin{aligned}
& \frac{\partial}{\partial r}\left(r^{2} B_{r}\right)=0 \\
& \frac{\partial}{\partial r}\left(r u B_{\omega}\right)=0
\end{aligned}
$$

These two equations can be integrated to give

$$
\begin{gathered}
B_{r} \propto r^{-2} \\
B_{\omega} \propto(r u)^{-1}
\end{gathered}
$$

which represent the spiral pattern of the solar magnetic field.

\section{The Poynting Vector}

The equation of energy conservation can be integrated once:

$$
N\left(m_{i} \frac{u^{2}}{2}+5 k T-\frac{m_{i} G M_{\odot}}{r}\right)+r^{2} q_{r}+r^{2} p_{r}=F
$$

Here $N=r^{2} n u=$ constant, $G$ is the gravitational constant, $M_{\odot}$ the mass of the sun, $q_{r}$ the radial component of the heat flux, and $p_{r}$ the radial component of the Poynting vector

$$
\mathbf{p}=\frac{c}{4 \pi} \mathbf{B} \times(\mathbf{u} \times \mathbf{B})
$$

In the right side of (5), $F$ is a constant. Thus equation (5) expresses the conservation of the total energy in the steady-state expansion of the solar wind. During the expansion process, energy can be converted from one form into another, but the total energy must be conserved.

The radial component of the Poynting vector is

$$
p_{r}=\frac{u B^{2} \sin ^{2} \phi}{4 \pi}
$$

Making use of (4), we can express the flow of magnetic field energy per steradian as

$$
r^{2} p_{r} \propto u^{-1}
$$

The solar wind velocity $u$ is an increasing function of the heliocentric distance $r$. Thus the flow of magnetic field energy per steradian is a decreasing function of $r$. Conservation of the total energy flow requires that the field energy be transformed into other forms of energy flow.

\section{Mechanism of Energy Conversion}

The equation of motion for the magnetohydrodynamic expansion of the solar wind is

$$
m_{i} n \frac{D \mathbf{u}}{D t}=m_{i} n \mathbf{g}-\nabla(2 n k T)+\frac{1}{c} \mathbf{J} \times \mathbf{B}
$$

where $\mathrm{g}=-\mathrm{e}_{r} G M_{\odot} / r^{2}$. In the right hand side of (7), the magnetic field exerts a force

$$
\frac{1}{c} \mathbf{J} \times \mathbf{B}
$$

on the moving solar wind flow. The radial component of this force can be written as

$$
\frac{B^{2} \sin ^{2} \phi}{4 \pi u} \frac{d u}{d r}
$$

This term is always positive, because $u$ is an increasing function of $r$. The magnetic field exerts a force on the solar wind in the direction of the solar wind velocity. 
The rate of work done by this force on a unit volume of * the solar wind flow is

$$
\frac{B^{2} \sin ^{2} \phi}{4 \pi} \frac{d u}{d r}
$$

The work done on the solar wind flow is used to increase the kinetic energy of the solar wind at the expense of the field energy.

\section{MAGNETOHYDRODYNAMIC ONE-FLUID MODEL OF THE SOLAR WIND}

\section{Governing Equations}

The magnetohydrodynamical expansion of the one-fluid solar wind is governed by a system of two ordinary nonlinear differential equations: The equation of motion

$$
\frac{d u}{d r}=\frac{u}{r} \frac{\frac{4 k T}{m_{i}}-\frac{G M_{\odot}}{r}-\frac{2 k r}{m_{i}} \frac{d T}{d r}}{u^{2}-\frac{2 k T}{m_{i}}-\frac{B^{2} \sin ^{2} \phi}{4 \pi m_{i} n}}
$$

and the equation of energy conservation

$$
\begin{aligned}
& r^{2} \kappa \cos ^{2} \phi \frac{d T}{d r} \\
& \quad=N\left(m_{i} \frac{u^{2}}{2}+5 k T-\frac{m_{i} G M_{\odot}}{r}+\frac{B^{2} \sin ^{2} \phi}{4 \pi n}\right)-F
\end{aligned}
$$

where the thermal conductivity $\kappa$ is proportional to $T^{5 / 2}$.

Let us denote conditions at the critical radius by a superscript asterisk. Then we can introduce the following dimensionless variables

$$
\begin{aligned}
& V=u / u^{*} \\
& \theta=T / T^{*} \\
& Z=r / r^{*}
\end{aligned}
$$

and dimensionless parameters

$$
\begin{aligned}
& \alpha=\frac{2 N k}{r^{*} \kappa^{*} \cos ^{2} \phi^{*}} \\
& \gamma=\frac{G M_{\odot}}{r^{*} u^{* 2}} \\
& H=\frac{F}{N m_{i} u^{* 2}}
\end{aligned}
$$

$$
\xi=\frac{m_{i} u^{* 2}}{2 k T^{*}}
$$

Because the denominator of (8) vanishes at the critical radius, we obtain

$$
\xi=1+\sin ^{2} \phi^{*} \frac{B^{* 2} / 8 \pi}{n^{*} k T^{*}}=\frac{1+2 \sin ^{2} \phi^{*}}{\beta^{*}}
$$

In terms of the dimensionless quantities, we can write the two governing equations, (8) and (9), in dimensionless form,

$$
\frac{d V}{d Z}=\frac{V}{Z} \frac{2 \theta-\frac{\gamma \xi}{Z}-Z \frac{d \theta}{d Z}}{\xi V^{2}-\theta-(\xi-1) \frac{1}{V}}
$$

and

$\frac{d \theta}{d Z}=\frac{\alpha}{Z^{2} \theta^{5 / 2}} \frac{\cos ^{2} \phi^{*}}{\cos ^{2} \phi}\left[\xi \frac{V^{2}}{2}+(\xi-1) \frac{1}{V}+\frac{5}{2} \theta-\frac{\gamma \xi}{Z}-\xi H\right]$

These two ordinary differential equations will suffice to solve for the two dependent variables $V$ and 0 .

At the critical radius, equation (11) reduces to

$$
\left(\frac{d \theta}{d Z}\right)^{*}=\alpha\left[\xi\left(\frac{3}{2}-\gamma-H\right)+\frac{3}{2}\right]
$$

Both the denominator and the numerator of equation (10) must vanish simultaneously at $Z=1$. When the numerator is zero, we obtain

$$
\left(\frac{d \theta}{d Z}\right)^{*}=2-\gamma \xi
$$

From the above two equations, we can show that $\alpha$ is not an independent parameter:

$$
\alpha=\frac{\gamma \xi-2}{\xi(\gamma+H-3 / 2)-3 / 2}
$$

Therefore, the general solutions for $V$ and $\theta$ can be expressed in the form

and

$$
\left.\begin{array}{l}
V=V\left(Z, H, \gamma, \xi, \phi^{*}\right) \\
\theta=\theta\left(Z, H, \gamma, \xi, \phi^{*}\right)
\end{array}\right\}
$$

Making use of the l'Hospital's rule, we can calculate $(d V / d Z)^{*}$; the result is 


$$
\left(\frac{d V}{d Z}\right)^{*}=-C_{1}+\left(C_{1}{ }^{2}+C_{2}\right)^{1 / 2}
$$

where

$$
\begin{aligned}
& C_{1}=\frac{\alpha+(\xi \gamma-2)\left(1+2 \sin ^{2} \phi^{*}\right)}{(6 \xi-2)} \\
& C_{2}=\frac{2+\alpha\left(\frac{3}{2} \xi \gamma-5\right)+(\xi \gamma-2)\left(\frac{5}{2} \xi \gamma+2 \sin ^{2} \phi^{*}-7\right)}{(3 \xi-1)}
\end{aligned}
$$

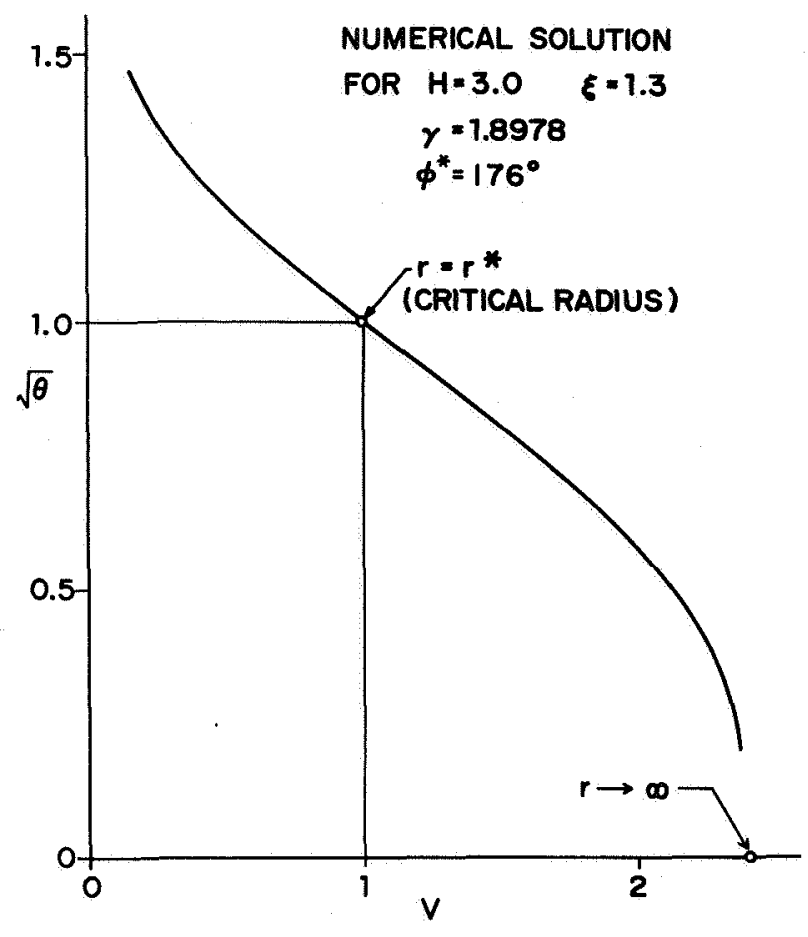

\section{Numerical Solutions}

For any given value of $\gamma, H, \xi$, and $\phi^{*}$, we can use (13) and (16) to calculate the solution for $V(Z)$ and $\theta(Z)$ in the $\epsilon$ neighborhood of the singular point at $Z=1$. Then we can integrate equations (10) and (11) for $Z>1+\epsilon$ and $Z<1-\epsilon$. The two branches of the numerical solutions join smoothly at the singular point.

The numerical solutions for $Z>1$ show the same property as the numerical solutions for the inviscid model of the solar wind [Whang and Chang, 1965]. As $Z \rightarrow \infty$, the solution curve is expected to approach the condition $V=V_{\infty}$ and $\theta=0$ with

$$
\frac{V_{\infty}^{2}}{2}+\frac{\xi-1}{\xi V_{\infty}}=H
$$

A solution satisfying this condition when $H=3.0$, $\xi=1.3, \phi^{*}=176^{\circ}$, and $\gamma=1.8978$ is obtained as shown in figure 1. The numerical values of this solution in dimensionless form $V=V(Z)$ and $\theta=\theta(Z)$ are given in table 1 .

Physical interpretation of the numerical solution depends on the choice of the ratio $r^{*} / r_{0}$. When the critical radius is chosen at between 5 and $6 r_{\odot}$, the solar wind conditions at $1 \mathrm{AU}$ predicted by the present solution are (fig. 2) $u=287-318 \mathrm{~km} / \mathrm{sec}$ and $T=(1.45-1.57) \times 10^{5} \mathrm{~K}$. In the remaining part of this paper, we will interpret the physical meaning of the solution based on $r^{*} / r_{\mathrm{e}}=5.5$ (fig. 3). The predicted solar wind conditions at $1 \mathrm{AU}$ are

$$
\begin{aligned}
u & =302 \mathrm{~km} / \mathrm{sec} \\
T & =1.50 \times 10^{5}{ }^{\circ} \mathrm{K} \\
\frac{d T}{d r} & =5.7 \times 10^{-9}{ }^{\circ} \mathrm{K} / \mathrm{cm} \\
\phi & =129.5^{\circ} \\
\beta & =1.58
\end{aligned}
$$

where $\beta$ is defined as the ratio of $2 n k T$ to $B^{2} / 8 \pi$.
Figure 1. The numerical solution, given in table 1, smoothly passes through the singular point at the critical radius $(Z=1)$, and approaches the condition $V=V_{\infty}$ and $\theta=0$ as $Z \rightarrow \infty$.

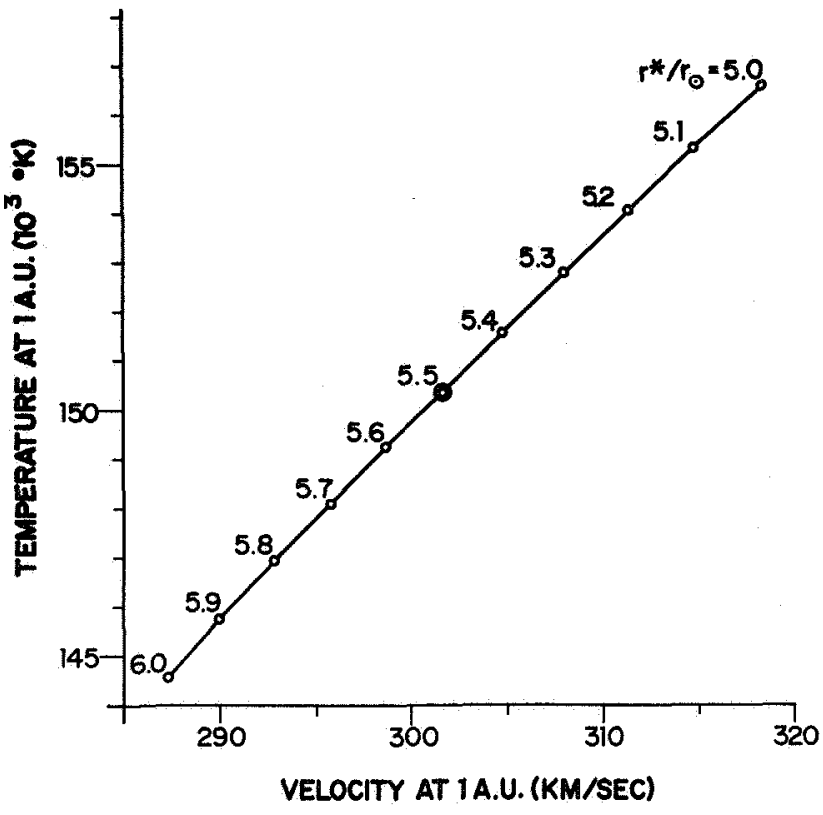

Figure 2. The solar wind conditions at 1 AU predicted by the present solution depend on the choice of the ratio $r^{*} / r_{\odot}$. For $r^{*} / r_{\odot}=5.5$, the present result gives $u=302 \mathrm{~km} / \mathrm{sec}$ and $T=1.50 \times 10^{5}{ }^{\circ} \mathrm{K}$ at $1 \mathrm{AU}$. 
Table 1. Numerical solution for $H=3.0, \xi=1.3, \phi^{*}=176^{\circ}$ and $\gamma=1.8978$

\begin{tabular}{|c|c|c|c|l|c|}
\hline$Z$ & $\phi$ & $V$ & $\theta$ & $d \theta / d Z$ & $\beta$ \\
\hline & & & & & \\
0.100 & 171.3 & 0.046 & 3.026 & -14.76 & 0.021 \\
0.126 & 172.7 & 0.069 & 2.704 & -10.53 & 0.020 \\
0.158 & 174.0 & 0.105 & 2.415 & -7.483 & 0.019 \\
0.200 & 175.0 & 0.159 & 2.157 & -5.294 & 0.017 \\
0.251 & 175.7 & 0.234 & 1.928 & -3.733 & 0.017 \\
& & & & & \\
0.316 & 176.2 & 0.331 & 1.724 & -2.630 & 0.017 \\
0.398 & 176.4 & 0.448 & 1.544 & -1.853 & 0.018 \\
0.501 & 176.5 & 0.579 & 1.384 & -1.309 & 0.019 \\
0.631 & 176.5 & 0.719 & 1.241 & -0.926 & 0.022 \\
0.794 & 176.3 & 0.860 & 1.114 & -0.657 & 0.027 \\
& & & & & \\
1.000 & 176.0 & 1.000 & 1.000 & -0.467 & 0.033 \\
1.259 & 175.6 & 1.134 & 0.898 & -0.333 & 0.041 \\
1.585 & 175.0 & 1.261 & 0.807 & -0.237 & 0.052 \\
1.995 & 174.2 & 1.380 & 0.725 & -0.169 & 0.067 \\
2.512 & 173.3 & 1.489 & 0.651 & -0.121 & 0.089 \\
& & & & & \\
3.162 & 172.1 & 1.590 & 0.585 & $-8.61 \times 10^{-2}$ & 0.118 \\
3.981 & 170.6 & 1.682 & 0.525 & -6.14 & 0.157 \\
5.012 & 168.8 & 1.766 & 0.472 & -4.38 & 0.211 \\
6.310 & 166.5 & 1.842 & 0.424 & -3.11 & 0.283 \\
7.944 & 163.8 & 1.911 & 0.381 & -2.22 & 0.378 \\
& & & & & \\
10.00 & 160.5 & 1.974 & 0.343 & -1.58 & 0.503 \\
12.59 & 156.6 & 2.030 & 0.308 & -1.13 & 0.661 \\
15.85 & 152.0 & 2.081 & 0.277 & $-8.10 \times 10^{-3}$ & 0.850 \\
19.95 & 146.7 & 2.127 & 0.249 & -5.88 & 1.063 \\
25.12 & 141.0 & 2.169 & 0.223 & -4.35 & 1.277 \\
& & & & & \\
31.62 & 134.9 & 2.207 & 0.199 & -3.27 & 1.464 \\
39.81 & 128.8 & 2.241 & 0.175 & -2.51 & 1.588 \\
50.12 & 122.9 & 2.271 & 0.152 & -1.96 & 1.626 \\
63.10 & 117.5 & 2.298 & 0.130 & -1.53 & 1.567 \\
79.44 & 112.7 & 2.322 & 0.108 & -1.18 & 1.424 \\
& & & & & \\
100.0 & 108.5 & 2.342 & 0.087 & $-8.78 \times 10^{-4}$ & 1.222 \\
125.9 & 105.0 & 2.359 & 0.068 & -6.16 & 0.997 \\
158.5 & 102.1 & 2.372 & 0.052 & -3.87 & 0.781 \\
\hline
\end{tabular}

The values of $u, T, d T / d r, \phi$, and $\beta$ calculated above are independent of the constant $K$ used in the expression for the thermal conductivity $k=K T^{5 / 2}$. However, the proton number density, the magnitude of the magnetic field and the heat flux at $1 \mathrm{AU}$ predicted by the present solution depend on the value of the constant $K$ (table 2). When the value of $K$ is about half of that used in the ordinary expression for the thermal conductivity of fully ionized hydrogen, the predicted $n, B$, and $q$ agree very well with the observed solar 

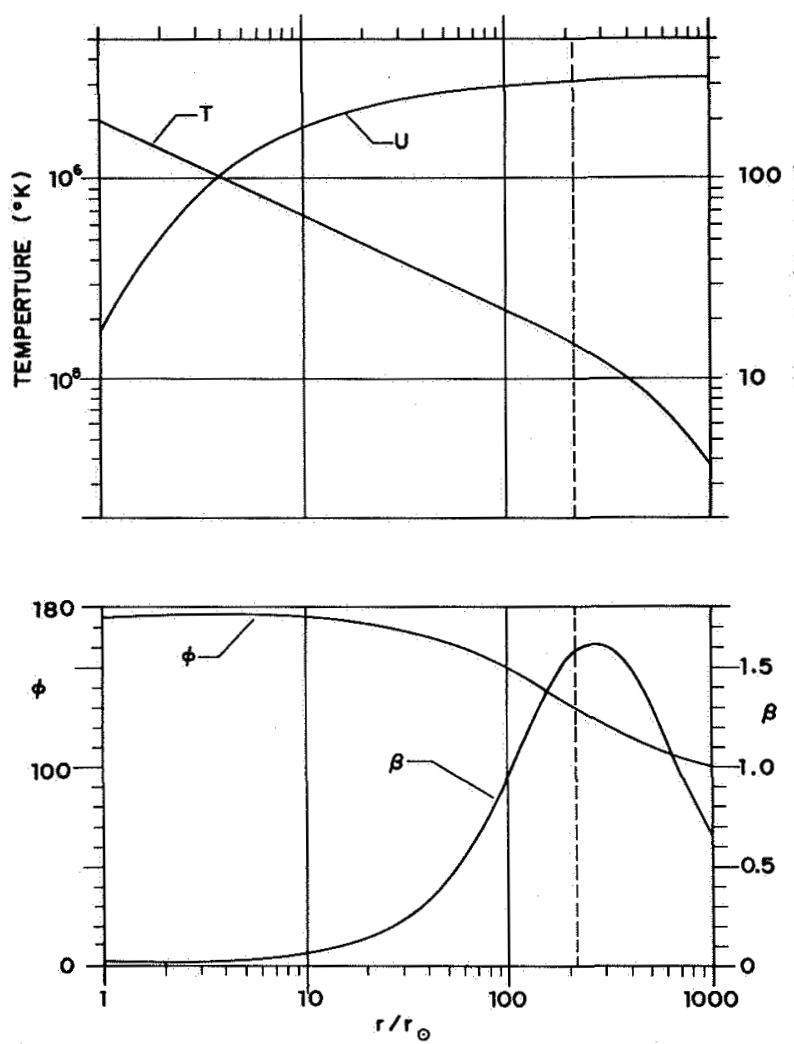

Figure 3. The solar wind temperature, velocity, direction angle, and $\beta$-value as functions of the heliocentric distance, at $1 \mathrm{AU} u=302 \mathrm{~km} / \mathrm{sec}, T=1.50 \times 10^{5}{ }^{\circ} \mathrm{K}$, $\phi=129.5^{\circ}$ and $\beta=1.58$.

Table 2. The proton density, the magnitude of the magnetic field, and the heat flux at $1 \mathrm{AU}$ predicted by the present solution

\begin{tabular}{cccc}
\hline $\begin{array}{c}k / T^{5 / 2}, \\
\text { ergs cm-1 } \\
\mathrm{sec}^{-1} \mathrm{deg}^{-7 / 2}\end{array}$ & $\begin{array}{c}n, \\
\text { protons } \\
\mathrm{cm}^{-3}\end{array}$ & $\begin{array}{c}B, \\
\text { gammas }\end{array}$ & $\begin{array}{c}q, \\
\text { ergs } \mathrm{cm}^{-2} \mathrm{sec}^{-1}\end{array}$ \\
\hline $6.00 \times 10^{-7}$ & 13.1 & 9.3 & $2.23 \times 10^{-2}$ \\
$4.58 \times 10^{-7}$ & 10.0 & 8.1 & $1.70 \times 10^{-2}$ \\
$4.00 \times 10^{-7}$ & 8.7 & 7.6 & $1.49 \times 10^{-2}$ \\
$3.66 \times 10^{-7}$ & 8.0 & 7.3 & $1.36 \times 10^{-2}$ \\
$2.75 \times 10^{-7}$ & 6.0 & 6.3 & $1.02 \times 10^{-2}$ \\
\hline
\end{tabular}

wind conditions [Hundhausen, 1970; Ness, 1967]. For $k=3.66 \times 10^{-7} T^{5 / 2} \mathrm{erg} \mathrm{cm} \mathrm{cm}^{-1} \mathrm{sec}^{-1} \mathrm{deg}^{-1}$, the predicted results at $1 \mathrm{AU}$ are $n=8$ protons $/ \mathrm{cm}^{3}$, $B=7.3 \gamma$ and $q=1.36 \times 10^{-2} \mathrm{erg} / \mathrm{cm}^{2} / \mathrm{sec}$. The predicted $n, B$, and $q$ as functions of the heliocentric distance $r$ are plotted in figures 4 and 5 . The density

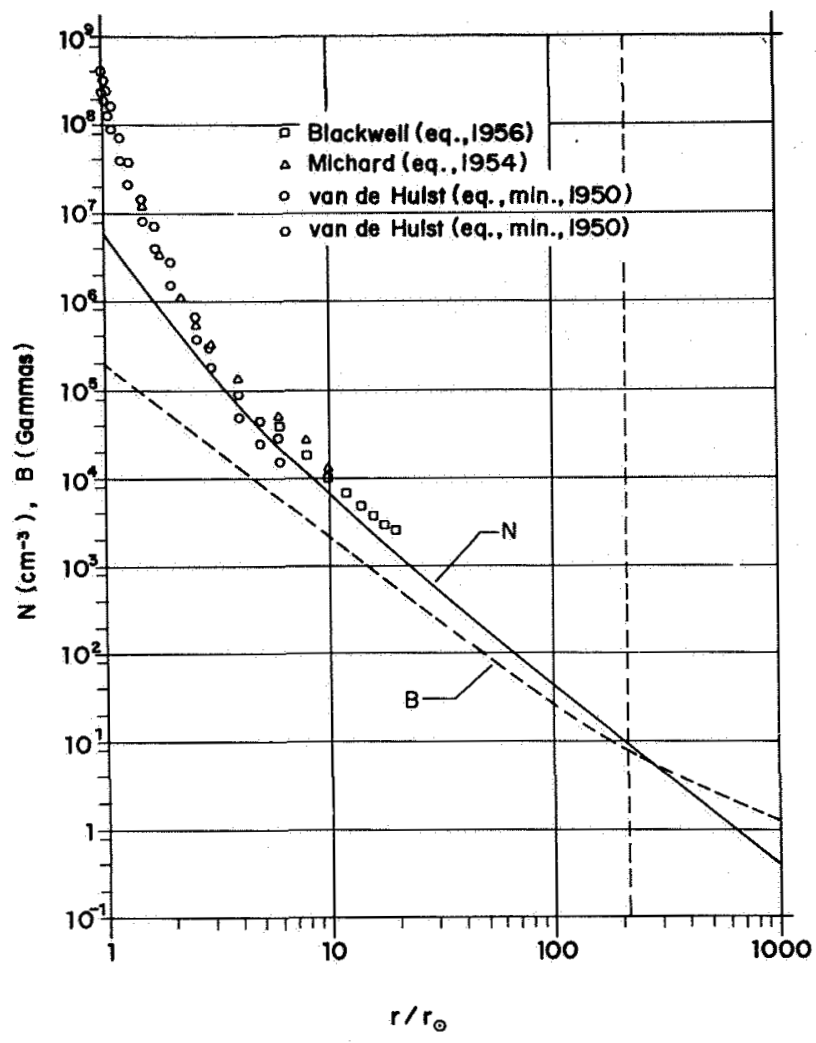

Figure 4. The proton density and the magnitude of the magnetic field as functions of the heliocentric distance.

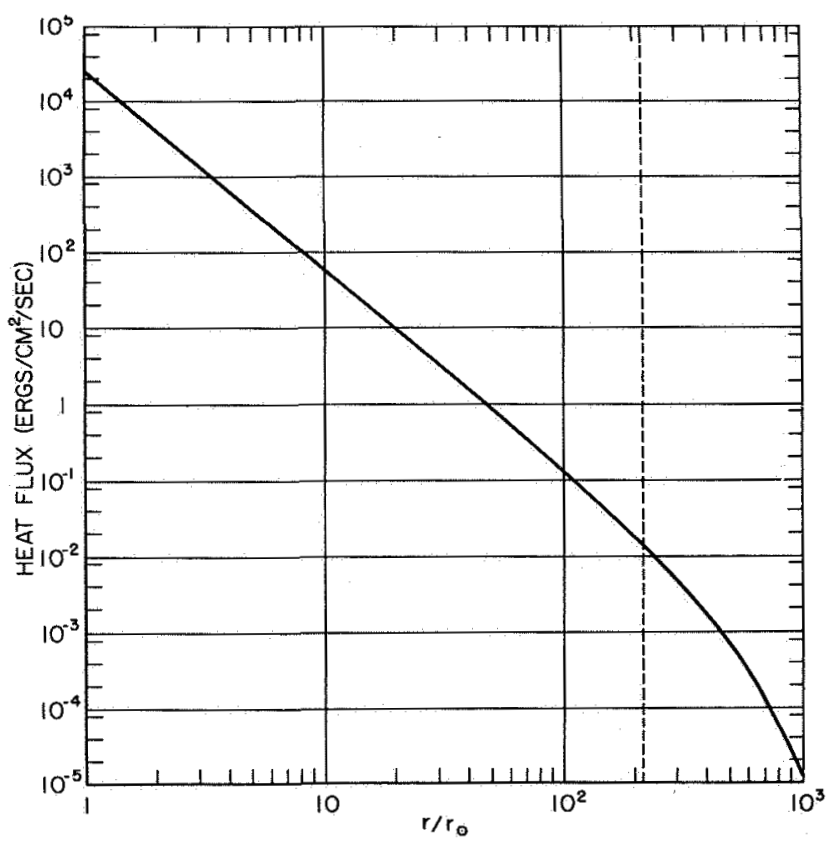

Figure 5. The heat flux as a function of the heliocentric distance, $q=1.4 \times 10^{-2} \mathrm{erg} / \mathrm{cm}^{2} / \mathrm{sec}$ at $1 \mathrm{AU}$. 
curve agrees reasonably well with the observed data in the region $2 r_{\odot}<r<20 r_{\odot}$ [van de Hulst, 1950, 1953; Michard, 1954; Blackwell, 1956]. In the region $r<2 r_{\odot}$, the present solution is not expected to be accurate, because the structure of the solar magnetic field is more complicated than the simple model described by equations (3) and (4).

\section{CONCLUDING REMARKS}

The solar wind conditions predicted by the present magnetohydrodynamic model show that when the effect of the solar magnetic field is included in the one-fluid model, we obtain an increase of 17 percent in the solar wind velocity or 37 percent in the convective kinetic energy at $1 \mathrm{AU}$. The variations of the magnetic field energy flow and the convective kinetic energy flow per steradian as functions of the heliocentric distance $r$ are plotted in figure 6 . The magnetic field energy flow per

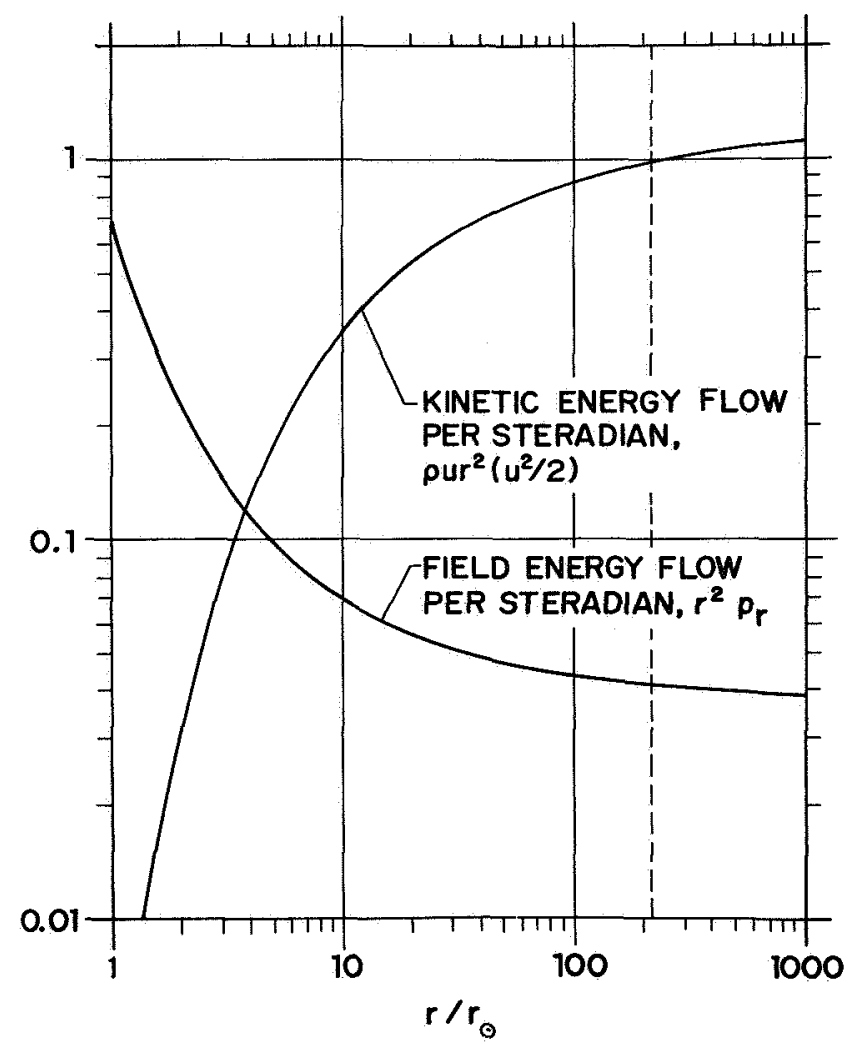

Figure 6. The radial flow of magnetic field energy per steradian is a decreasing function of the heliocentric distance. The field energy is converted into the kinetic energy during the expansion of the solar wind. The convective kinetic energy flow per steradian at $1 \mathrm{AU}$ is chosen as unity in this figure. steradian is a monotonically decreasing function of $r$. Using the convective kinetic energy per steradian at $1 \mathrm{AU}$ as a unit, we can see that the total decrease of the magnetic field energy flow from $r=2 r_{\odot}$ to $r=1 \mathrm{AU}$ is about 0.35 . Conversion of the magnetic field energy into the kinetic energy certainly plays an important role in the expansion process of the solar wind.

Urch [1969] has studied the magnetohydrodynamic one-fluid model of the solar wind. The equations of motion and energy conservation were integrated in a quite different way. He has obtained the numerical solutions for the solar wind after several iterations. The temperatures at $1 \mathrm{AU}$ predicted by Urch are 3 to $4 \times 10^{5} \mathrm{~K}$, which are too high compared with the observed data.

Recently Wolff et al. [1971] have studied the magnetohydrodynamic two-fluid model of the solar wind; their predicted solar wind conditions at $1 \mathrm{AU}$ are very close to the results obtained in this paper. Barnes et al. [1971] have also included the magnetic field in their two-fluid model of the solar wind. Because they have dropped out the $\mathbf{J} \times \mathbf{B} / c$ force term in their equation of motion, the magnetic field force is not doing work on the expanding solar wind. The conversion of magnetic field energy into kinetic energy is not included in their work. They proposed that an energy source due to dissipation of hydromagnetic waves supplies the energy needed to increase the solar wind velocity. Their source of energy is completely different from the magnetic field energy flow (the Poynting vector) discussed here.

\section{ACKNOWLEDGMENTS}

This work was supported by National Aeronautics and Space Administration under Grant No. NGR-09-005-063.

\section{REFERENCES}

Barnes, A.: Collisionless Heating of the Solar Wind Plasma, I, Theory of Heating of a Collisionless Plasma by Hydromagnetic Waves. Astrophys. J., Vol. 154, 1968, p. 751 .

Barnes, A.: Collisionless Heating of the Solar Wind Plasma, II, Application of the Theory of Plasma Heating by Hydromagnetic Waves. Astrophys. J., Vol. 155,1969 , p. 311.

Barnes, A.; Hartle, R. E.; and Bredekamp, J. H.: On the Energy Transport in Stellar Winds. Astrophys. $J$. Ltrs., Vol. 166, 1971, p. L-53.

Blackwell, D. E.: A Study of the Outer Solar Corona from a High Altitude Aircraft at Eclipse of 1954, June 30. Mon. Not. Roy. Astron. Soc., Vol. 116, 1956, p. 57. 
Hartle, R. E.; and Sturrock, P. A.: Two-Fluid Model of the Solar Wind. Astrophys. J., Vol. 151, 1968, p. 1155 .

Hundhausen, A. J.: Composition and Dynamics of the Solar Wind Plasma. Rev. Geophys. Space Phys., Vol. 8,1970 , p. 729.

Jokipii, J..R.; and Davis, L. Jr.: Long Wavelength Turbulence and the Heating of the Solar Wind. Astrophys. J., Vol. 156, 1969, p. 1101.

Michard, R: Densitie's E'lectroniques Dans la Couronne Externe du 25 Février 1952. Ann. Astrophys., Vol. 17,1954, p. 429.

Ness, N. F.: Observed Properties of the Interplanetary Plasma. Ann. Rev. Astron. Astrophys., Vol. 6, 1967, p. 79.

Noble, L. M.; and Scarf, F. L.: Conductive Heating of the Solar Wind, I. Astrophys. J., Vol. 138, 1963, p. 1169.

Parker, E. N.: Dynamics of the Interplanetary Gas and Magnetic Fields. Astrophys. J., Vol. 128, 1958, p. 664 .
Sturrock, P.A.; and Hartle, R. E.: Two-Fluid Model of the Solar Wind. Phys. Rev. Letters, Vol. 16, 1966, p. 628 .

Urch, I. H.: A Model of the Magnetized Solar Wind. Solar Phys., Vol. 10, 1969, p. 219.

Whang, Y.C.; and Chang, C. C.: An Inviscid Model of the Solar Wind. J. Geophys. Res., Vol. 70, 1965, p. 4175.

Wolff, C. L.; Brandt, J. C.; and Southwick, R. G.: A Two Component Model of the Quiet Solar Wind with Viscosity, Magnetic Field, and Reduced Heat Conduction. Astrophys. J., Vol. 164, Pt. I, 1971, p. 181 .

van de Hulst, H.C.: The Electron Density of the Solar Corona. Bull. Astron. Inst. Neth., Vol. 11, 1950, p. 136.

van de Hulst, H. C.: The Chromosphere and the Corona, in The Sun, edited by G.P. Kuiper, Univ. of Chicago Press, Chicago, 1953, p. 259. 